\title{
Clinical Analysis of Primary Hepatic Neuroendocrine Tumors: Presentation of a Case Report with Intraoperative Carcinoid Crisis and Review of Current Literature
}

\author{
Wojciech M Korcz ${ }^{1}$, Paweł Nyckowski ${ }^{2}$, Gustaw Lech ${ }^{3}$, Michał Mazurkiewicz ${ }^{4}$, Alicja Kwiatkowska ${ }^{5}$, Maciej Słodkowski ${ }^{6}$
}

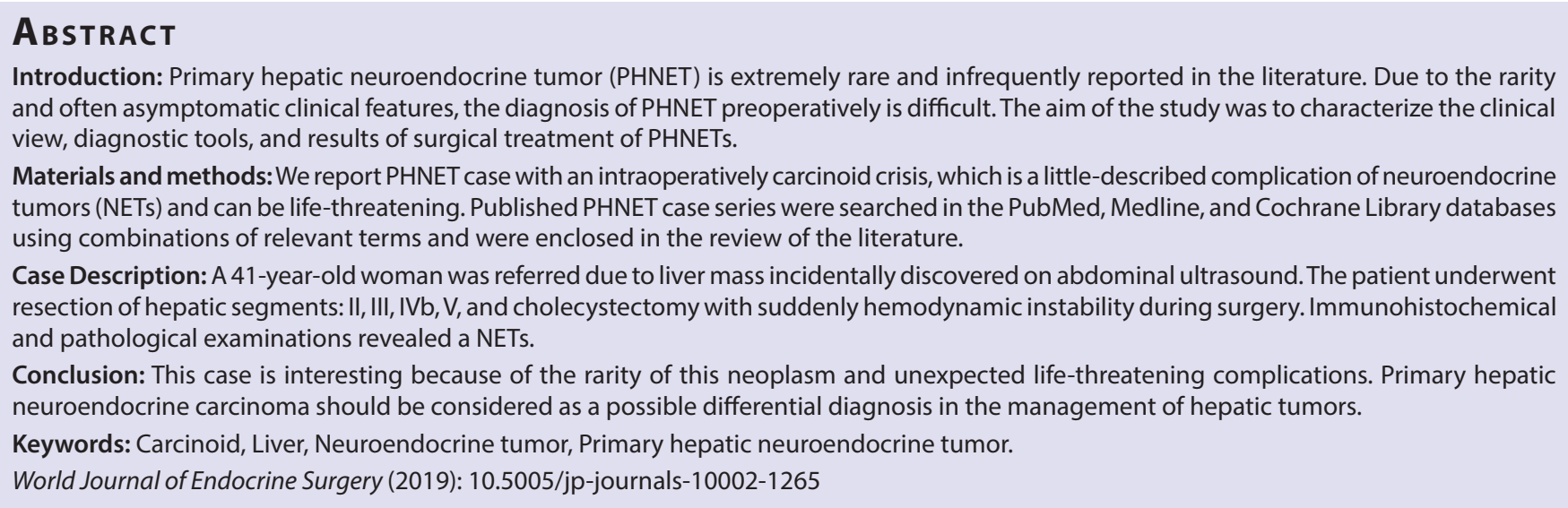

\section{INTRODUCTION}

Neuroendocrine tumors (NETs) develop most often along the gastrointestinal tract (54-75\%), especially in the small intestines (35-45\%) and rectum (19.6-23\%). ${ }^{1-8}$ Primary hepatic NETs (PHNETs), also known as carcinoid tumors, although extremely rare, increasingly receive the attention of clinicians. Tumors arise from scattering neuroendocrine cells in the epithelium of the intrahepatic biliary tract or heterotopic tissue located in the liver. ${ }^{2,4,9}$ Primary hepatic NETs represent only $0.3-5.5 \%$ of all neuroendocrine malignancies and are reported about $1-2 \%$ of all gastrointestinal tumors. ${ }^{3,4,6,8,10-16}$ Most of the hepatic NETs (about 54-90\%) are metastases from gastroenteropancreatic NETs, especially from pancreas (30\%) and rectum (17\%)..$^{5,10-13,15,17-19}$ Due to the rarity of PHNETs, clinical features, treatment outcomes, and tumor characteristic dates are poorly described. There are usually low-grade tumors, which can secrete hormones like chromogranin $\mathrm{A}(\mathrm{CgA})$, serotonin, and gastrin. ${ }^{2}$ Due to rarity, it is a diagnostic and therapeutic challenge to make the correct diagnosis. In this article, we present a case report of a patient with PHNET who underwent liver resection complicated by severe, intraoperative, and hemodynamic instability. Articles to review of the literature were selected from the PubMed, Medline, and Cochrane databases using the following keywords: primary hepatic neuroendocrine tumor, neuroendocrine tumor, liver, and carcinoid crisis. We mainly focused on the original, full-text case series. The aim of the study was to present the current view on clinical features, treatment, diagnostic tools, and outcomes of surgical treatment of PHNETs.

\section{Case Description}

A 41-year-old female patient was admitted to the Department of General and Oncological Surgery of the Central Teaching Hospital of
1-3,6 Department of General, Gastroenterological and Oncological Surgery, Medical University of Warsaw, Warsaw, Poland

${ }^{4}$ Department of Pathology, Medical University of Warsaw, Warsaw, Poland

${ }^{5}$ Department of Anesthesiology and Intensive Therapy, Medical University of Warsaw, Warsaw, Poland

Corresponding Author: Wojciech M Korcz, Department of General, Gastroenterological and Oncological Surgery, Medical University of Warsaw, Warsaw, Poland, Phone: +48 22599 2258, e-mail: wojtek. korcz@interia.pl

How to cite this article: Korcz WM, Nyckowski P, Lech G, et al. Clinical Analysis of Primary Hepatic Neuroendocrine Tumors: Presentation of a Case Report with Intraoperative Carcinoid Crisis and Review of Current Literature. World J Endoc Surg 2019;11(3):94-99.

Source of support: Nil

Conflict of interest: None

Medical University with, "a symptomatic hepatic mass" founded by ultrasound 1 month earlier and confirmed on magnetic resonance image (MRI). Ultrasound examination revealed a heterogeneous, solid, hyperechoic tumor located in the left lobar, with diameter $60 \times 46 \mathrm{~mm}$. Magnetic resonance image showed heterogeneous, well-circumscribed liver masses in segment IV $(40 \times 43 \mathrm{~mm})$ with low intensity in T1-weighted images and high intensity on T2-weighted images. The patient referred only abdominal pain in the right epigastrium for 6 months without other clinical symptoms. The routine laboratory studies abnormalities apart from a slight elevation of liver blood test: alanine aminotransferase $57 \mathrm{U} / \mathrm{L}$ (normal 7-56 U/L), aspartate aminotransferase $88 \mathrm{U} / \mathrm{L}$ (normal 5-40 U/L), and lactate dehydrogenase $264 \mathrm{U} / \mathrm{L}$ (normal 135-225 U/L). Serum tumor 
markers, such as a-fetoprotein (AFP), carcinoembryonic antigen (CEA), carbohydrate antigen 19-9 (Ca19-9) were within the normal range and negative serology for hepatitis. The patient was qualified for surgical treatment. Intraoperatively tumor in segment III, IVb, and $V$ of the liver was found. At the beginning of liver resection, which was started in the area of segment $V$, there was a sudden severe hypotension and flushing, without bronchospasm. There was no obvious cause of hypotension-neither tumor manipulation nor using drugs that stimulate the sympathomimetic system or lead to histamine release. Systolic pressures were ranged from 40 to almost $0 \mathrm{~mm} \mathrm{Hg}$. Initial management included intravenous bolus of ephedrine $(10 \mathrm{mg}+5 \mathrm{mg}$ ) with crystalloid bolus $(20 \mathrm{~mL} / \mathrm{kg})$. Due to a lack of response to this management, it started to continuous intravenous adrenaline infusion (range from 0.02 to $0.08 \mu \mathrm{g} / \mathrm{kg}$ / minute) and norepinephrine infusion (range from 0.02 to $0.08 \mu \mathrm{g} /$ $\mathrm{kg} /$ minute). Emergency echocardiography was performed during surgery-without any abnormalities and causes of hemodynamic instability. In total, $4,000 \mathrm{~mL}$ of fluid and $1 \mathrm{mg}$ of adrenaline was administered. Furthermore, the patient received a single-shot high dose of methylprednisolone (125 mg) and clemastine (2 mg). After hemodynamic stabilization (blood pressure 110/60 $\mathrm{mm} \mathrm{Hg}$ ) liver resection has been continued. The patient underwent resection of hepatic segments II, III, IVb, V, and cholecystectomy was performed. At the intensive care unit, the patient did not require any vasopressor support. After surgery, the patient remained intubated due to tongue swelling. She was recovered and successfully extubated on the next day. The resected specimen weighed $350 \mathrm{~g}$ and solid, white and well-circumscribe tumor in segment IVb/V measured $4 \mathrm{~cm}$ in diameter was found. Pathological and immunohistochemical examinations (Fig. 1) revealed neuroendocrine carcinoma with no complete excision surgical margins (R1 resection); with hemorrhagic and necrotic areas. Multifocal angioinvasion with vascular thrombosis was observed. Tumor cells were positive for the following markers: synaptophysin (Syn), CgA, and CD19, by immunohistochemical studies. The Ki-67 index was $30 \%$ and the mitotic count was 10/10 per high-power field. She was discharged home on day 7 after surgery in a good general condition. Postoperative work-up, including the thorax and abdomen computerized tomography (CT), MRI, PET-CT with 68Ga, PET-CT with ${ }^{18} \mathrm{~F}$-fluorodeoxyglucose, panendoscopy excluded the presence of extrahepatic primary neuroendocrine neoplasm or metastasis. 2 months after surgery, ${ }^{18} \mathrm{~F}$-fluorodeoxyglucose PET imaging revealed high FDG (fluorodeoxyglucose) uptake in the liver segment IVa (diameter $12 \mathrm{~mm}$ ). Magnetic resonance image was performed 3 months after primary surgery-revealed cystic lesion located laterally and below segment IVa liver measuring $88 \times$ $28 \mathrm{~mm}$ and perfusion disorders at the area of the unresected part of the liver segment IVa (Fig. 2). The last MRI images, performed 8 months after primary surgery, showed 3 lesions with maximum diameter up to $15 \mathrm{~mm}$, located on the surface of liver segments VII and VIII. After performing a systematic examination and follow-up of 8 months to exclude metastasis or extrahepatic primary tumor, we diagnosed a PHNET.

\section{Discussion/Review of Literature}

Several previous studies and our review of the literature indicate that PHNETs mostly occur in middle-aged patients, with the mean age range from 43.7 years to 66.5 years - $^{2-10-13,17-20}$ and no clear gender preference exist (female $25.71-66.67 \%$ vs male $33.33-$ $74.92 \%) .^{1-4,9-13,16-20}$ Size of malignancies ranged from $0.6 \mathrm{~cm}$ to even
$27 \mathrm{~cm}$ and the majority of tumors located on the right lobe of the liver $37.5-49.3 \%{ }^{1-3,8,9,11,16,17}$ The average diameter equaled $3.82-6.73 \mathrm{~cm}$ or even measured up to $8.6 .^{2,3,11,16,17}$ Most tumors (>90\%) were $>3 \mathrm{~cm}$ in diameter at the moment of diagnosis. ${ }^{19}$ The masses were observed more often unilobar (61.67-81.5\%) than bilobar (18.5-38.33\%), and more frequent there were single nodule $(55.6-76.6) .^{1-4,8,10-13,16,17,20}$ Table 1 presents the clinical data review of patients with PHNETs from the original articles. Clinical manifestations of PHNET are nonspecific. Various sources report that $69.51-73.4 \%$ of patients were symptomatic and the most common symptom of PHNET was abdominal pain, which can affect as many as $33.3-77 \%$ of all cases (Table 2 ). ${ }^{1-4,9-13,16,17,20}$ Carcinoid syndrome can occur in 3.66-18.9\% of patients. ${ }^{1-4,6,9-13,16,17,19,20}$ Many patients are discovered by routine screening or health examination with a solid tumor mass. ${ }^{2,12}$ It is also noteworthy, even 23.2-41\% patients did not have any presenting symptoms. ${ }^{2-4,9-13,16,20}$ Diagnosis of PHNET is challenging and it is difficult to differentiate preoperatively from other tumors. Usually, serologic tumor markers such as AFP, CEA, or Ca19-9 are within normal limits. ${ }^{13,16}$ Serum analysis of CgA (sensitivity of $87-100 \%$, specificity $84-95 \%$ ) and 5 -hydroxyindoleacetic acid (5-HiAA) in 24-hour urine collection (sensitivity $74 \%$, specificity $90-96 \%$ ) should be assessed during diagnosis process of NETs and can help detect tumor recurrence. ${ }^{5-7,9,20}$ Serum CgA level can be false-positive in patients with hepatic or renal failure, chronic proton pump inhibitor. It is important to keep in mind to not mistake carcinoid tumor with hepatocellular carcinoma (HCC). ${ }^{3,16,20}$ On images, PHNET is detected as hypervascular (in the arterial phase), heterogeneous, well-demarcated solid tumor mass with or without cystic changes, accompanied by satellite hypodense nodules. $2,3,6,12,13,18-20$ Primary hepatic NETs had a rich blood supply from the hepatic artery, which can contribute to misdiagnosis as HCC. Computed tomography images PHNETs show low-density mass on precontrast phase with diffuse heterogeneous enhancement, on MRI-low intensity in T1-weighted images, and high intensity on T2-weighed images. ${ }^{2,6,17-21}$ Endoscopy scanning, somatostatin receptor scintigraphy and positron emission tomography (PET) scans are also performed for detection of primary tumor. ${ }^{8}$ Positron emission tomography scan can detect tumor as small as $2 \mathrm{~mm}$ in diameter and PHNETs often has high ${ }^{18} \mathrm{~F}$-fluoro-deoxyglucose uptake. ${ }^{2,6,10,20}$ Researchers suggested that octreotide scintigraphy is useful in detecting the primary tumors, recurrence or exclude extrahepatic liver tumors with the sensitivity of $80-90 \% .6,7,9,10,12,18,20$ It has the highest specificity for NETs-83-88\% show somatostatin receptors, which can be used to predict response to octreotide analog treatment. ${ }^{6,13,15}$ It should be a routine preoperative approach of suspected NETs because it even identifies an additional $16 \%$ of distant tumors, which are invisible for CT or MRI. ${ }^{15}$ There is no standard definition of carcinoid crisis-it contains both severe diarrhea and unexpected fluctuating blood pressure during surgical manipulation., ${ }^{42}$ Carcinoid tumors can cause unpredictable circulation complications, which are results of the release of neurosecretory products into the systemic circulation. According to guidelines for care in NETs, treatment with long-acting somatostatin analogs can contribute to control and inhibit the excessive hormonal output and prevent carcinoid crisis. ${ }^{23}$ Clinicians must keep in mind that high CgA levels, high urinary 5-HIAA, large tumor mass are risk factors for developing an intraoperative carcinoid crisis. The ideal dosing method of octreotide has not been published yet, but PHNETs guidelines recommend the administration of octreotide $100 \mu \mathrm{g}$ three times/day subcutaneously for 2 weeks before intra-abdominal surgery. ${ }^{23-25}$ In emergency cases, we could administer additional, an intravenous bolus of $500 \mu$ g. $^{5}$ Some authors suggested perioperative 



Fig. 1A to D: (A) Tumor cells were strongly immunoreactive for chromogranin A (immunohistochemistry, magnification $\times 100)$; (B) Immunoreactivity for CK19 in the tumor cells (immunohistochemistry, magnification $\times 100)$; (C) Infiltration of neuroendocrine cancer cells (hematoxylin-eosin staining, magnification $\times 40)$; (D) Infiltration of neuroendocrine cancer cells (hematoxylin-eosin staining, magnification $\times 100$ )

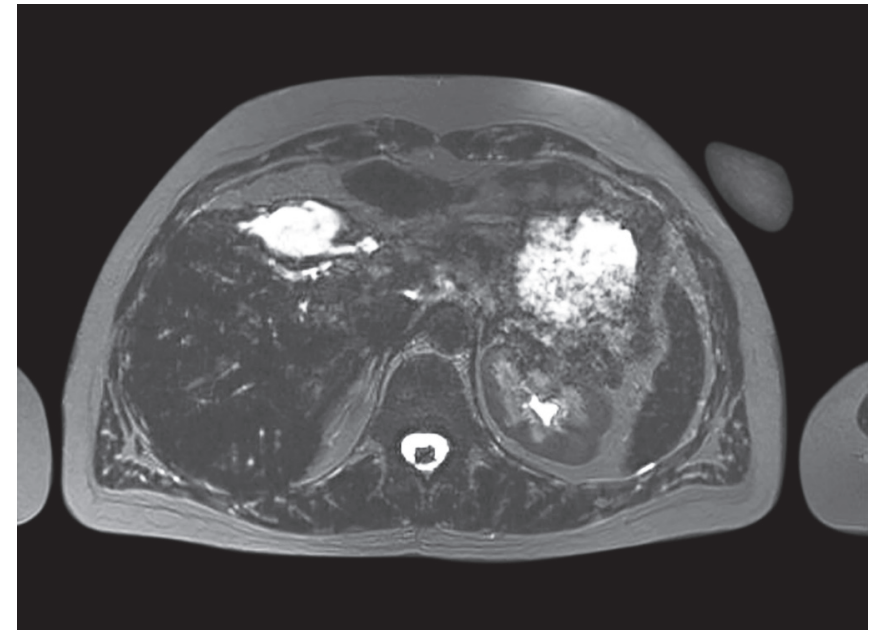

Fig. 2: Magnetic resonance image. T2-weighted axial image, showing fluid collection and hepatic perfusion disorders (segment IVa)

prophylactic treatment with intravenous octreotide, which started 12 hours before and routinely continued at least 48 hours after surgery with the initial mean dose $100-200 \mu \mathrm{g} /$ hour. ${ }^{5,23,25}$ About $11-15 \%$ of patients who did not receive octreotide analogs before an operation, suffered from carcinoid crisis vs $0-3.4 \%$ which had prophylaxis. $4,5,12,22,23$ Surgery is the most effective treatment and it is recommended as the first option of treatment for PHNETs and liver resection can be carried out in about $85 \%$ of cases. ${ }^{1,4,9,10,12,15,16,19,20}$ It should pay attention to the fact that a definitive preoperative diagnosis may be delayed due to asymptomatic and often nonspecific clinical presentation. The recurrence rate of PHNETs is high: 18-40\%, 5-years after surgical resection, while some authors suggest that this rate is even $70 \%$ (range from 6 months to 15 years). ${ }^{1,3-5,10,11,19,20}$ Multiple tumors experienced more frequent recurrences than those with solitary tumors 40 vs $15 \% .^{5}$ Patients treated by major resection experienced significantly fewer frequent recurrences than those by subsegmental resection (17 vs $59 \% p$ value: 0.017 ). ${ }^{4}$ Huang at all revealed the 1-year recurrence rate was $45.5 \% .{ }^{20}$ It was reported that $10-17 \%$ of cases develop metastases, so long-term follow-up is req uired. ${ }^{1,3,4,11,16}$ Patients with PHNETs demonstrated more tendency to have a regional lymph node invasion compared with metastatic hepatic NETs (28.57\% vs $8.7 \%) .{ }^{16}$ The mean interval from resection to recurrence was $36.6-48$ months. ${ }^{1,4}$ Notably, neuroendocrine cells diffused in the liver so it can explain the frequency of recurrences and often multifocal origin. ${ }^{4}$ Even $23.7-44.4 \%$ of patients had more than one tumor. ${ }^{1-4,10-13,16,17,20}$ Survival period after recurrence ranges from 3 months to 12 months. ${ }^{19}$ The 1-, 5-, 10-year, and overall survival rates for primary tumors were $95-75 \%, 56-80 \%$, and $50-68 \%$, 


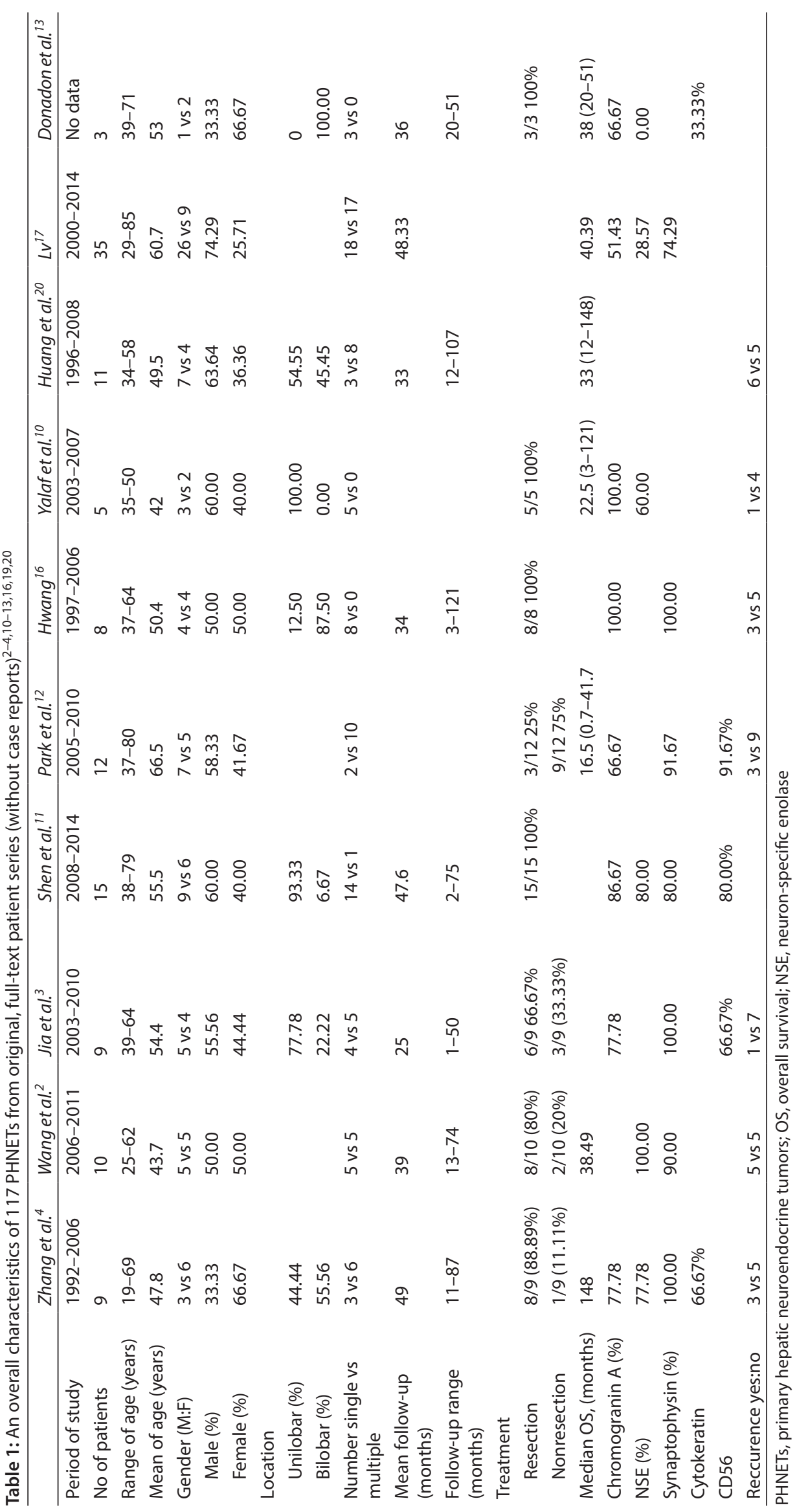


Table 2: Clinical presentation of patients with PHNETs from published articles $^{2-4,10-13,16,19,20}$

\begin{tabular}{lllll}
\hline Symptoms & \multicolumn{2}{c}{ (\%) } & \multicolumn{2}{c}{ Quartey $^{1}$ (\%) } \\
\hline Symptomatic & $57 / 82$ & 69.51 & $88 / 120$ & 73.3 \\
Asymptomatic & $25 / 82$ & 30.49 & $32 / 120$ & 26.7 \\
Abdominal pain & $40 / 82$ & 48.78 & $57 / 88$ & 26.7 \\
Abdominal mass & $1 / 82$ & 1.22 & $11 / 88$ & 12.5 \\
Jaundince & $2 / 82$ & 2.44 & $4 / 88$ & 4.5 \\
Body weight loss & $5 / 82$ & 6.1 & - & \\
Carcinoid syndrome & $3 / 82$ & 3.66 & $6 / 88$ & 6.8 \\
Other symptoms & $14 / 82$ & 17.07 & - & \\
\hline
\end{tabular}

PHNETs, primary hepatic neuroendocrine tumors

respectively, with a mean survival 148 months for resected primary tumors. ${ }^{2,4,5,9-11,13,17,20}$ Quartey analyzed 124 cases of PHNETs and revealed that $28.8 \%$ died after a mean follow-up of 41 months. ${ }^{1}$ Disease-free survival rate (DFS) was defined as the period from the initial date of treatment of PHNET to the date of the tumor recurrence or death. Massive researchers reported 1-, 5-, and 10-year DFS in patients who underwent radical surgical procedure is relatively favorable outcomes and estimated at 100-98, 80-74-30, and 75-68\%, respectively. ${ }^{2-5,10,11,15-17,20,25} 1-, 5-$, and 10-year DFS for unresected tumor equal 75,33 , and $0 \%$, respectively. ${ }^{2,4}$ Yang et al. revealed that mean DFS for PHNETs equal 24.92 months. ${ }^{17}$ There were no statistical significant differences in survival rates between patients with solitary and multiple tumors and between patients who underwent surgical resection for unilobar disease and bilobar disease. ${ }^{4}$ Multivariate analysis of the prognostic factors associated with overall survive by Yang et al. revealed that tumor character (soft/hard; $p=0.001$ ), number of tumors (solitary/multifocal; $p=0.043$ ), the immunohistochemical expression of neuron-specific enolase (NSE, $p=0.000)$ and Syn $(p=0.037)$ were statistically significant. ${ }^{17}$ However, both patients with primary $(n=35)$ and metastatic $(n=46)$ hepatic NETs were included in the analysis. ${ }^{[17]}$ Analysis by Hwang showed that the Ki-67 proliferative index was a significant tumor recurrence factor in univariate analysis and the average Ki-67 index in cases without recurrence was $1.7 \% .^{15,18}$ The 1-, 3-, and 5-year recurrence rates were 25,40 , and $18-40 \%$, respectively. ${ }^{16,20}$ Diagnosis is based on traditional histopathologyaccompanied by immunohistochemistry examination (PHNETs-released positivity for markers, such as NSE, Syn, and $(g A)$ with the postoperative exclusion of extrahepatic primary tumor. ${ }^{2,3,10,12,15,16}$ Long-time follow-up and postoperative diagnostic processes are necessary to exclude metastatic disease from an unknown primary origin., ${ }^{1,3,10,13}$ Careful investigation using many kinds of diagnostic methods (CT, MRI, scintigraphy, PET, panendoscopy, and bronchoscopy) is necessary to detect primary tumor. Syn and NSE confirm the neurosecretory character and show high specificity as markers for NETs., ${ }^{2,10}$ Primary hepatic NETs were positive for CgA (70.83-94.7\%), Syn (50.5-79.6\%), cytokeratin (13.7$58.33 \%)$, and NSE (48.05\%), respectively. ${ }^{1-4,10-13,16,17,20}$

\section{Conclusion}

Due to the rarity of PHNET, proper diagnosis compares with appropriate treatment is a challenge. Avoiding misdiagnosis from other solid tumors, especially HCC is important. Clinicians must keep in mind to remember about a high recurrence rate and long-term follow-up in order to exclude an underlying extrahepatic primary tumor. Correctly diagnose can guarantee good-long-term results.
Earl hypervascular, solitary tumor in imaging studies in the patient with normal serum AFP levels and without chronic liver disease should be suspected as PHNET.

\section{References}

1. Quartey B. Primary hepatic neuroendocrine tumor: what do we know now? World J Oncol 2011;2(5):209-216. DOI: 10.4021/wjon341w.

2. Wang LM, An SL, Wu JX. Diagnosis and therapy of primary hepatic neuroendocrine carcinoma: clinical analysis of 10 cases. Asian Pac J Cancer Prev 2014;15(6):2541-2546. DOI: 10.7314/APJCP.2014.15.6.2541.

3. Jia C, Zhang Y, Xu J, et al. Experience in primary hepatic neuroendocrine tumor. Turk J Gastroenterol 2012;23(5):546-551. DOI: 10.4318/ tjg.2012.0370.

4. Zhang A, Xiang J, Zhang $M$, et al. Primary hepatic carcinoid tumors: clinical features with an emphasis on carcinoid syndrome and recurrence. J Int Med Res 2008;36(4):848-859. DOI: $10.1177 / 147323000803600428$.

5. Castillo J, Silvay G, Weiner M. Anesthetic management of patients with carcinoid syndrome and carcinoid heart disease: the mount Sinai algorithm. J Cardiothorac Vasc Anesth 2018;32(2):1023-1031. DOI: 10.1053/j.jvca.2017.11.027.

6. Yang K, Cheng YS, Yang JJ, et al. Primary hepatic neuroendocrine tumors: multi-modal imaging features with pathological correlations. Cancer Imaging 2017;17(1):20. DOI: 10.1186/s40644-017-0120-x.

7. Yang K, Cheng YS, Yang JJ, et al. Primary hepatic neuroendocrine tumor with multiple liver metastases: a case report with review of the literature. World J Gastroenterol 2015;21(10):3132-3138. DOI: 10.3748/ wjg.v21.i10.3132.

8. Song JE, Kim BS, Lee CH. Primary hepatic neuroendocrine tumor: a case report and literature review. World J Clin Cases 2016;4(8):243-247. DOI: 10.12998/wjcc.v4.i8.243.

9. Gravante G, De Liguori Carino N, Overton J, et al. Primary carcinoids of the liver: a review of symptoms, diagnosis and treatments. Dig Surg 2008;25(5):364-368. DOI: 10.1159/000167021.

10. Yalaf $O$, Ülku A, Akcam TA, et al. Primary hepatic neuroendocrine tumor: five cases with different preoperative diagnoses. Turk J Gastroenterol 2012;23(3):272-278. DOI: 10.4318/tjg.2012.0465.

11. Shen YH, Chen S, Zhang WT, et al. Clinical analysis of gastroenteropancreatic neuroendocrine tumor with liver metastasis, compared with primary hepatic neuroendocrine tumor. J Cancer Res Ther 2014;10:276-280. DOI: 10.4103/0973-1482.151532.

12. Park CH, Chung JW, Jang SJ, et al. Clinical features and outcomes of primary hepatic neuroendocrine carcinomas. J Gastroenterol Hepatol 2012;27(8):1306-1311. DOI: 10.1111/j.1440-1746.2012.07117.x.

13. Donadon M, Torzilli G, Palmisano A, et al. Liver resection for primary hepatic neuroendocrine tumours: report of three cases and review of the literature. EJSO 2006;32(3):325-328. DOI: 10.1016/ j.ejso.2005.11.017.

14. Knox CD, Anderson CD, Lamps LW, et al. Long-term survival after resection for primary hepatic carcinoid tumor. Ann Surg Oncol 2003;10(10):1171-1175. DOI: 10.1245/ASO.2003.04.533.

15. DeLuzio MR, Barbieri AL, Israel G, et al. Two cases of primary hepatic neuroendocrine tumors and a review of the current literature. Ann Hepatol 2017;16(4):621-629. DOI: 10.5604/01.3001.0010.0313.

16. Hwang $S$, Lee YJ, Lee SG, et al. Surgical treatment of primary neuroendocrine tumorsnof the liver. J Gastrointest Surg 2008;12: 725-730. DOI: 10.1007/s11605-007-0418-2.

17. Lv $\mathrm{Y}$, Huang $\mathrm{C}, \mathrm{Xu} \mathrm{H}$, et al. Clinicopathological characteristics of the primary and metastatic hepatic neuroendocrine tumors and the relevant prognosis-related factors: a retrospective study of 81 cases in a single chinese center. J Cancer 2018;9(3): 479-487. DOI: 10.7150/jca.22157.

18. Lambrescu IM, Martin S, Cima L, et al. Primary hepatic neuroendocrine tumor after 4 years tumor-free follow-up. J Gastrointestin Liver Dis 2015;24(2):241-244. DOI: 10.15403/jgld.2014.1121.242.yrs.

19. Lin CW, Lai CH, Hsu CC, et al. Primary hepatic carcinoid tumor: a case report and review of the literature. Cases J 2009;2(1):90. DOI: $10.1186 / 1757-1626-2-90$. 
20. Huang YQ, Xu F, Yang JM, et al. Primary hepatic neuroendocrine carcinoma: clinical analysis of 11 cases. Hepatobiliary Pancreat Dis Int 2010;9(1):44-48.

21. Chen $Z$, Xiao HE, Ramchandra $P$, et al. Imaging and pathological features of primary hepatic neuroendocrine carcinoma: an analysis of nine cases and review of the literature. Oncol Lett 2014;7(4):956-962. DOI: 10.3892/ol.2014.1844.

22. Condron ME, Pommier SJ, Pommier RF. Continuous infusion of octreotide combined with perioperative octreotide bolus does not prevent intraoperative carcinoid crisis. Surgery 2016;159(1):358-365. DOI: 10.1016/j.surg.2015.05.036.
23. Kaltsas G, Caplin M, Davies P, et al. Antibes consensus conference participants. ENETS consensus guidelines for the standards of care in neuroendocrine tumors: pre- and perioperative therapy in patients with neuroendocrine tumors. Neuroendocrinology 2017;105(3): 245-254. DOI: 10.1159/000461583.

24. Dierdorf SF. Carcinoid tumor and carcinoid syndrome. Curr Opin Anaesthesiol 2003;16(3):343-347. DOI: 10.1097/00001503-20030600000017.

25. Mancuso K, Kaye AD, Boudreaux JP, et al. Carcinoid syndrome and perioperative anesthetic considerations. J Clin Anesth 2011;23(4): 329-341. DOI: 10.1016/j.jclinane.2010.12.009. 